\title{
Gastric ulcers: malignancy yield and risk stratification for follow-up endoscopy
}

Authors

Institutions
Christian P Selinger ${ }^{1,2}$, Rebecca Cochrane ${ }^{1}$, Sangeetha Thanaraj ${ }^{1}$, Anita Sainsbury ${ }^{1}$, Venkat Subramanian ${ }^{1,2}$, Simon Everett ${ }^{1}$

${ }^{1}$ Department of Gastroenterology, Leeds Teaching Hospitals NHS Trust, Leeds, UK

${ }^{2}$ University of Leeds, Leeds, UK submitted 7. October 2015 accepted after revision 11. April 2016

\section{Bibliography}

Dol http://dx.doi.org/ 10.1055/s-0042-106959 Published online: 19.5.2016 Endoscopy International Open 2016; 04: E709-E714

(c) Georg Thieme Verlag KG Stuttgart · New York E-ISSN 2196-9736

\section{Corresponding author} Dr Christian Selinger Gastroenterology

Leeds Teaching Hospitals NHS Trust

Beckett Lane

Leeds

LS9 7TF

United Kingdom

Phone: +44 1132068768

Fax: +44 1132068851

Christian.selinger@web.de
Background and study aim: Malignant change can occur in gastric ulcer but guideline recommendations for follow-endoscopy (FU-OGD) are conflicting. This study aims to determine rate of malignancy and need for follow-up for gastric ulcers. Patients and methods: Patients with a first diagnosis of gastric ulcer between January 2012 and September 2013 were studied by analyzing endoscopic assessments, dysplasia, and malignancy yield and the influence of risk factors on the likelihood of benign disease.

Results: In a cohort of 432 patients with gastric ulcer (53\% male, mean age 65 years) dysplasia or neoplasia were found in 27 (19 adenocarcinomas, 2 cases of dysplasia, 5 lymphomas, 1 melanoma; malignancy yield 6\%). Twenty-five (93\%) cases were diagnosed on first biopsy. The cancer yield of FU-OGD after initially benign biopsy was $0.9 \%$. Binary logistic regression analysis revealed that endoscopically benign appearance (odds ratio

\section{Introduction}

\section{$\nabla$}

Gastric ulceration is a common worldwide problem which can often lead to dyspeptic symptoms, epigastric pain, gastrointestinal bleeding and/or anemia. While Helicobacter pylori is commonly implicated in the pathogenesis of benign ulceration [1], the risk of malignant change in gastric ulcer has long been recognized [2]. In the literature, the rate of malignancy in endoscopically diagnosed gastric ulcers varies considerably, ranging from $2.4-21 \%[3,4]$.

Subsequently there has been disagreement over the requirements for follow-up in patients with gastric ulcers. A number of studies have reported low rates of additional gastric cancer diagnoses when following up patients with gastric ulcers $[3,5]$. This was especially the case if initial histology showed benign features and if no endoscopic suspicion was raised [5].
$0.00495 \%$ CI $0-0.576 ; P=0.029)$, benign histology on first biopsy (odds ratio $095 \% \mathrm{CI} 0-0.39$; $P=$ 0.011 ) and lower number of ulcers (odds ratio 0.22 (95\% CI $0.05-0.99$ ); $P=0.049$ ) were independent predictors of benign disease. All dysplastic and neoplastic cases would have been identified by a combination of initial biopsies plus repeat endoscopy with further biopsies for endoscopically suspicious appearances.

Conclusions: In this large cohort $6 \%$ of gastric ulcers were found to be malignant, highlighting the need for all gastric ulcers to be biopsied. The cancer yield of FU-OGD after benign biopsies was low. We have demonstrated that the combination of benign index histology and no endoscopic suspicion of malignancy can predict benign disease. We recommend that all gastric ulcers to be biopsied. Risk stratification could potentially reduce need for FU-OGD.

The discrepancy in study findings and researchers' opinions has also translated into international guidelines. The British National Institute for Health and Care Excellence (NICE) recommends a follow-up endoscopy (FU-OGD) for every patient with a gastric ulcer 8 weeks after index endoscopy $[6,7]$. In contrast, the American Society for Gastrointestinal Endoscopy recommends followup endoscopy only for selected cases of gastric ulcers [8]. Neither guideline advises on the need for biopsies of gastric ulcers at index endoscopy or follow-up.

The aim of this study was to determine the malignancy yield in gastric ulcers in a British setting, assess the impact of NICE guidance, and establish predictive factors potentially allowing a more targeted follow-up program. 


\section{Patients and methods}

$\nabla$

Leeds Teaching Hospitals NHS Trust is the main secondary care provider for a population of 770,000. The endoscopy department operates 10 endoscopy rooms at 3 sites performing approximately 20,000 procedures per annum.

For this study we searched gastroscopy records within the electronic endoscopy reporting system (ADAM, Fujifilm Europe $\mathrm{GmbH}, 2011$ ) and included all patients with a first endoscopic diagnosis of gastric ulcer between January 2012 and September 2013. Patients with known gastric ulcers prior to the study period or those referred for tertiary assessment were excluded. Patient demographics, data on endoscopic appearance and histological reports were recorded from ADAM and the electronic patient record system. Endoscopy procedures were performed by a group of certified endoscopists comprising gastroenterologists, surgeons, trainees, and nurse endoscopists. Gastric ulcers were defined as a mucosal break of $5 \mathrm{~mm}$ or larger in diameter with unequivocal depth, and erosion by mucosal change with white necrotic substance [9].

The primary analysis of the whole study cohort included analysis of endoscopic assessments, compliance with NICE guidance, and the overall combined dysplasia and malignancy yield. To assess compliance with required service standards we analyzed the proportion of FU-OGD performed within 8 weeks of index endoscopy (national standard) and the number of FU-OGD performed within 2 weeks of index endoscopy if no biopsies were taken (locally agreed standard).

For the secondary analysis of predictors of benign and malignant disease, we excluded patients with insufficient follow-up to ensure that no patient with a potential malignant transformation within 1 year was classified as benign disease. Insufficient follow-up was therefore defined as the lack of follow-up endoscopy and lack of data within the medical records to ascertain cancerfree survival of at least 360 days. Neoplastic disease was defined as histologic evidence of gastric dysplasia or malignancy.

Benign disease was defined as patients with complete ulcer healing, those with at least 2 sets of benign biopsies and no endoscopic suspicion of malignant disease, or in cases without FUOGD, 1 set of negative biopsies and at least 360 days cancer-free survival. Patients with insufficient follow-up (as judged by the above criteria) were excluded from the secondary analysis.

Endoscopic ulcer appearance was recorded from the endoscopy reports. Ulcers were classified as superficial, cratered or linear, which are the preset classification criteria on the endoscopy reporting system. The endoscopist's subjective impression was recorded dichotomously (suspicious appearance/non-suspicious appearance) by analysing the free text comment on the endoscopy reports (if suspicious features were not documented the ulcer was classed as showing "non-suspicious appearance").

We analyzed the influence of demographic, endoscopic, and histologic factors on the likelihood of benign disease using chisquare test for categorical and t-test for continuous variables. Independence of variables was analyzed by entering variables showing statistical significance on univariate analysis into binary logistic regression analysis. The Hosmer-Lemeshow's test was used to test the null hypothesis that there was a linear relationship between predictor variable and the log odds of the outcome variable. Correlation matrices were used to identify collinearity. If collinearity was detected we planned to minimize this by inputting the variable separately in the multivariate analysis. All statistical tests were done using PASW version 21 (IBM Corp, NY).

\begin{tabular}{|c|c|}
\hline Age & $\begin{array}{l}\text { Mean } 65 \text { years } \\
46 \text { aged } \leq 40 \text { years } \\
386 \text { aged }>40 \text { years }\end{array}$ \\
\hline Sex & $\begin{array}{l}229 \text { male }(53 \%) \\
203 \text { female }(47 \%)\end{array}$ \\
\hline Presenting features & $\begin{array}{l}164 \text { gastrointestinal bleed }(38 \%) \\
56 \text { anaemia }(13 \%) \\
78 \text { dyspepsia }(18 \%) \\
134 \text { other symptoms ( } 31 \%)\end{array}$ \\
\hline First biopsy & $\begin{array}{l}290 \text { on index endoscopy (mean } 4.8 \text { samples) } \\
142 \text { patients not biopsied initially } \\
-73 \% \text { gastrointestinal bleed } \\
-6 \% \text { anticoagulation } \\
-21 \% \text { no reason }\end{array}$ \\
\hline $\begin{array}{l}\text { Number of ulcers on } \\
\text { index endoscopy }\end{array}$ & $\begin{array}{l}1 \text { ulcer: } 289 \text { patients }(67 \%) \\
2 \text { ulcers: } 39 \text { patients }(9 \%) \\
3 \text { ulcers: } 26 \text { patients }(6 \%) \\
\text { More than } 3 \text { ulcers: } 78 \text { patients }(18 \%)\end{array}$ \\
\hline $\begin{array}{l}\text { Size of largest ulcer on } \\
\text { index endoscopy }\end{array}$ & $\begin{array}{l}\text { Mean size } 11 \mathrm{~mm} \\
148 \text { patients with largest ulcer } \geq 10 \mathrm{~mm} \\
284 \text { patients with largest ulcer }<10 \mathrm{~mm}\end{array}$ \\
\hline $\begin{array}{l}\text { Morphology of ulcers } \\
\text { on index endoscopy }\end{array}$ & $\begin{array}{l}\text { Superficial: } 172 \text { patients }(49 \%) \\
\text { Cratered: } 162 \text { patients ( } 38 \%) \\
\text { Other/not documented: } 98 \text { patients ( } 23 \% \text { ) }\end{array}$ \\
\hline Ulcer location & $\begin{array}{l}\text { Antrum: } 251 \text { patients ( } 58 \%) \\
\text { Body: } 75 \text { patients }(17 \%) \\
\text { Fundus / GOJ: } 46 \text { patients ( } 11 \%) \\
\text { Other / not documented: } 60 \text { patients ( } 14 \%)\end{array}$ \\
\hline $\begin{array}{l}\text { Helicobacter pylori } \\
\text { status }\end{array}$ & $\begin{array}{l}\text { Positive } 78(18 \%) \\
\text { Negative } 202(47 \%) \\
\text { Not tested for } 152(35 \%)\end{array}$ \\
\hline $\begin{array}{l}\text { Endoscopically suspi- } \\
\text { cious appearance }\end{array}$ & $\begin{array}{l}\text { Yes: } 33 \text { patients }(8 \%) \\
\text { No: } 399 \text { patients }(92 \%)\end{array}$ \\
\hline
\end{tabular}

The study was performed as a clinical audit of routinely collected patient data. Clinical audits are exempt from the need of ethics committee approval and the need for written informed consent under UK regulations [10].

\section{Results}

\section{$\nabla$}

The primary analysis cohort consisted of 432 patients ( $53 \%$ male) with a mean age of 65 years ( $\bullet$ Table 1 ). The presenting feature leading to endoscopy was a gastrointestinal bleed in $38 \%$, anaemia in $13 \%$, dyspepsia in $18 \%$ and other symptoms (including weight loss or any combination of symptoms) in $31 \%$.

No biopsies were taken in 142 patients at index endoscopy as $73 \%$ of these had presented with a gastrointestinal bleed ( $\bullet$ Fig. 1 ) and $6 \%$ were fully anticoagulated at the time of endoscopy. In $21 \%$ no reason to withhold biopsies was given. Of these $142,67 \%$ (96 patients) received a FU-OGD for biopsies after a median of 7 days (range 1 - 363 days, interquartile range 3 - 38 days). No FUOGD occurred in 46 of these cases.

Two hundred and ninety patients had an average of 4.8 biopsies taken on index endoscopy. Of these, 194 underwent FU-OGD after a median of 70 days (range 3-639 days, interquartile range 52 - 108 days). Only $33 \%$ underwent FU-OGD within the recommended time frame of 8 weeks. Reasons for not undergoing FUOGD included failure to request the procedure by the medical team in $10 \%$. A repeat procedure was deemed medically inappropriate (comorbidities, life expectancy, etc) by the clinical team in a further $19 \%$. Twelve percent of patients died before 


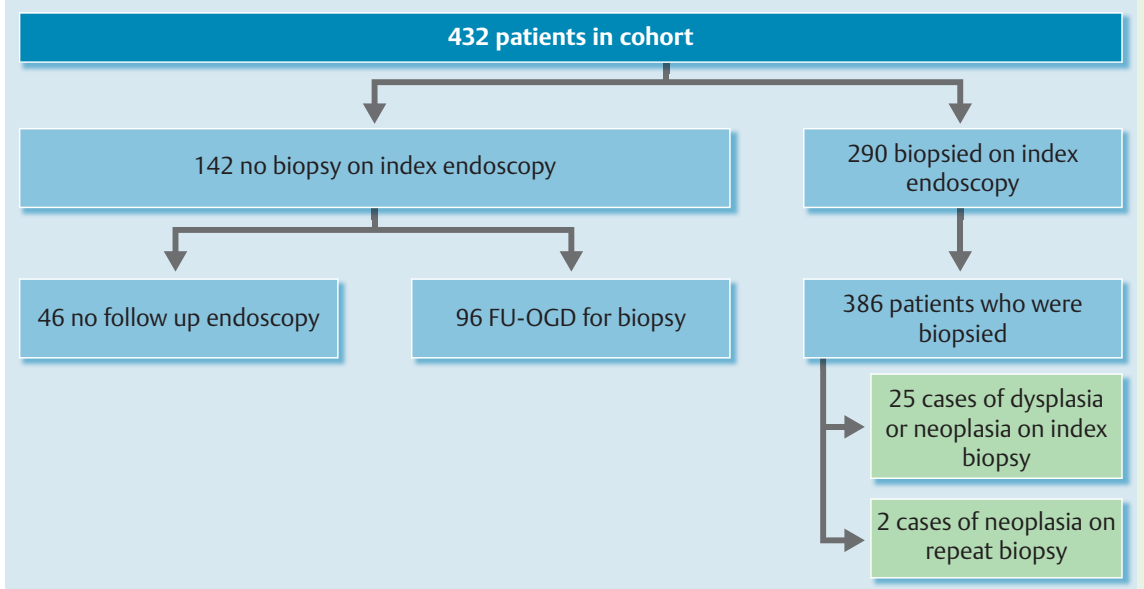

Fig. 1 Patient flow in the study cohort.

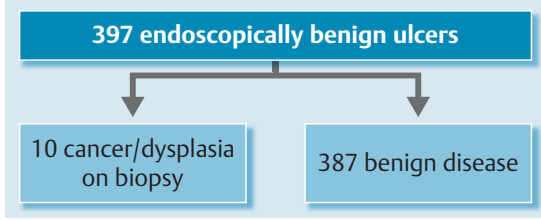

35 endoscopically suspicious ulcers

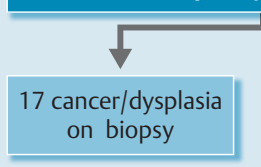

Fig. 2 Outcomes stratified by endoscopic and histologic appearance.

Table 2 Predictors of benign disease.

\begin{tabular}{|llll} 
Predictor & Univariate analysis & $\begin{array}{l}\text { Multivariate analysis Odds ratio (logistic } \\
\text { regression analysis) with } 95 \% \mathrm{Cl}\end{array}$ & $\begin{array}{l}P \text { value (logistic regression } \\
\text { analysis) }\end{array}$ \\
\hline Ulcer location in the antrum & $P=0.001$ & $0.1(0.003-3.228)$ & $P=0.194$ \\
\hline Endoscopically benign appearance & $P<0.001$ & $0.004(0-0.576)$ & $P=0.029$ \\
\hline First biopsies benign & $P<0.001$ & $0.0(0.00-0.39)$ & $P=0.011$ \\
\hline Younger age (mean) & 64 vs 73 years, $P=0.02$ & $1.039(0.938-1.150)$ & $P=0.046$ \\
\hline Lower number of ulcers (mean) & 1.4 vs 2 & $0.22(0.05-0.99)$ & $P=0.802$ \\
\hline Smaller ulcer size (mean) & $P<0.001$ & $0.992(0.933-1.055)$ & \\
\hline
\end{tabular}

the FU-OGD was due. Other reasons for a lack of FU-OGD included patient non-attendance (24\%) and malignant disease on first biopsy (9\%). There was no recorded reason in $26 \%$.

FU-OGD occurred overall in 290 patients. Complete ulcer healing was documented endoscopically in 236 patients. Gastric neoplasia was found in 27 patients ( 19 adenocarcinomas, 2 cases of lowgrade dysplasia [11], 5 lymphomas, 1 melanoma) leading to a diagnosis of malignancy in $6 \%$ of patients with gastric ulcers ( Fig. 1). Of these, 25 (93\%) were diagnosed on first biopsy. Three hundred and fifty-eight patients were at least biopsied once during the study. After excluding patients with dysplasia or malignancy on first biopsy $(n=25)$, without biopsies on repeat endoscopy ( $\mathrm{n}=28)$, and those without sufficient follow-up and/ or no further endoscopy after index biopsy $(n=80)$, the cancer yield of FU-OGD after initially benign biopsy was 2 of 225 patients $(0.9 \%)$. No cases of gastric cancer were detected in the group of patients without FU-OGD.

After excluding those patients with insufficient follow-up (as outlined in the methods section) the cohort for the secondary analysis consisted of 377 patients. Of these, 350 (92\%) had benign disease and 27 had dysplastic or neoplastic disease. Patient sex, indication for gastroscopy, and $H$ pylori status did not influence the likelihood of benign disease. Benign disease was significantly associated ( Table 2 ) with ulcer location in the antrum $(P=$ 0.001 ), endoscopic benign appearance as judged by the endos- copist at the time of procedure $(\bullet$ Fig. $2, P<0.001)$, non-cratered ulcer morphology ( Fig. 3, $P<0.001$ ), benign histology on first biopsy $(P<0.001)$, younger age (mean 64 vs 73 years, $P=0.02$ ), lower number of ulcers (mean 1.4 vs $2, P<0.001$ ), and smaller ulcer size (mean 10 vs $28 \mathrm{~mm}, P<0.001$ ). The number of biopsies taken was not associated with the likelihood of benign or malignant disease.

After binary logistic regression analysis, endoscopic benign appearance (odds ratio $0.00495 \% \mathrm{CI} 0-0.576 ; P=0.029$ ), benign histology on first biopsy (odds ratio $095 \% \mathrm{CI} 0-0.39 ; P=0.011$ ), and lower number of ulcers (odds ratio 0.22 (95\% CI $0.05-0.99$ ); $P=0.049$ ) were independent predictors of benign disease ( $\square \mathrm{Ta}$ ble 2). To rule out selection bias by including patients without FU-OGD, these patients were compared to those with FU-OGD and benign disease. Those without FU-OGD had significantly fewer biopsies (mean 4.59 vs $5.14 ; P=0.023$ ) and were more likely to have presented with "other symptoms" rather than a gastrointestinal bleed $(P=0.001)$. No differences in age, number of ulcers, ulcer size, location, or macroscopic or histologic appearance were found. No significant collinearity was detected on correlation matrices and the Hosmer-Lemeshows test was not significant, suggesting that the data fit the model well.

All dysplastic and neoplastic cases would have been correctly identified if a combination of at least 1 set of biopsies for all ulcers and repeat endoscopy with further biopsies for patients with ul- 

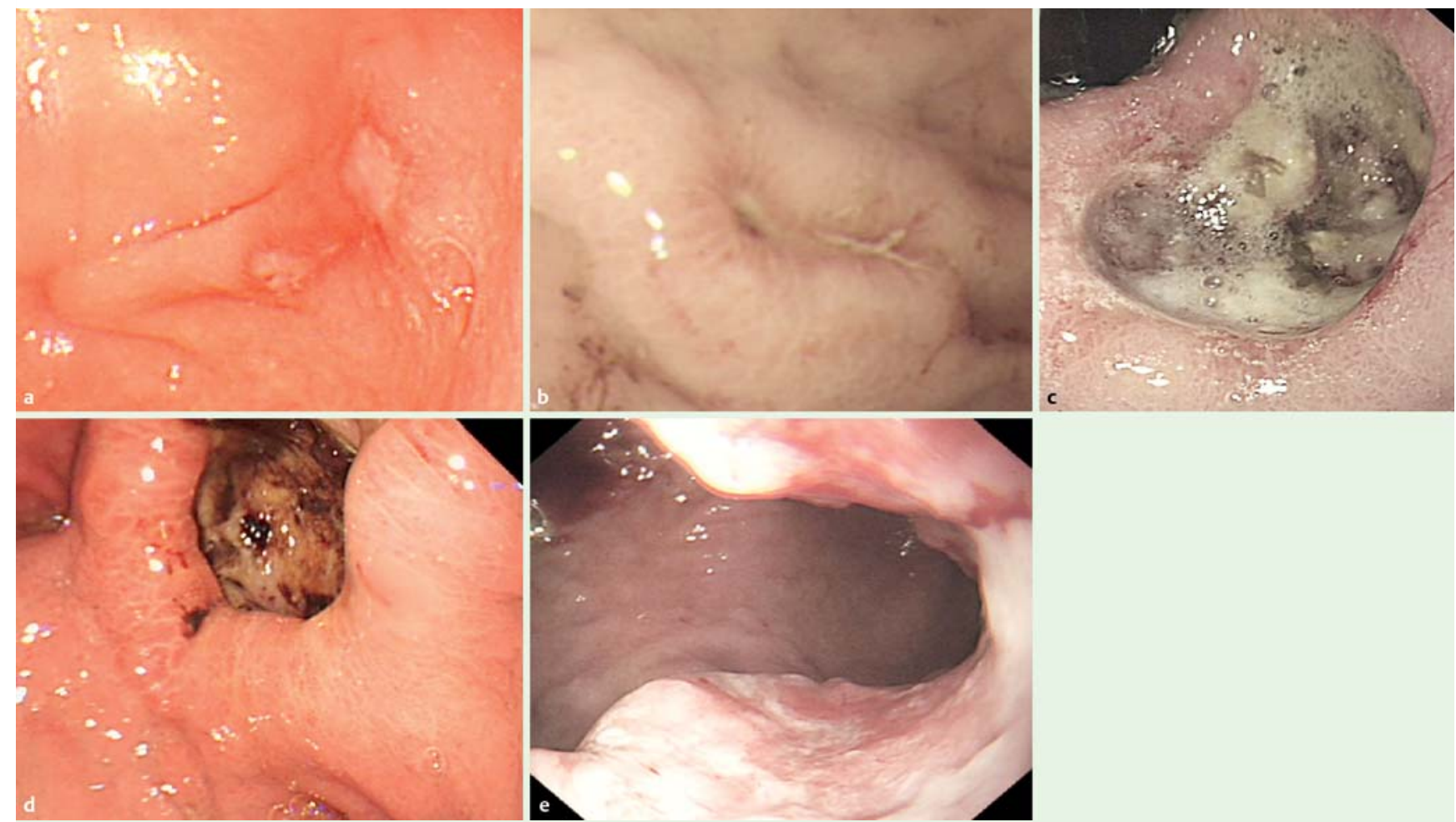

Fig. 3 Ulcer morphology. a Superficial ulcers. b Linear ulcer. c Cratered ulcer. $\mathbf{d}$ Rolled edges and mucosal irregularity. e Rolled edges and protrusion into the lumen.

cers displaying suspicious features on index endoscopy had been applied.

\section{Discussion \\ $\nabla$}

Medical opinion and international guidelines on the appropriate follow-up for patients with gastric ulcers remain divided. While British guidelines mandate FU-OGD for all patients [6,7], the American position of the ASGE advises that it is only required in selected cases [8]. In this large cohort of patients, it was demonstrated that gastric ulcers are associated with a significant risk of dysplasia or malignancy of $6 \%$. The major strength of this study is the non-selected secondary care cohort of patients thus reducing selection bias, which may explain the wide variation of malignancy rates reported in previously published case series. The large size of the cohort also allows for accurate determination of factors predicting benign or malignant disease. Furthermore, the endoscopies were performed by a diverse group of endoscopists rather than purely tertiary referral expert endoscopists.

The need for biopsies of all gastric ulcers has so far not been recognized by international guidelines. In this study $90 \%$ of cases of dysplasia or neoplasia were diagnosed with first biopsy, highlighting the need for all gastric ulcers to be biopsied. This is especially important as a number of ulcers with neoplasia on histology did not show endoscopically suspicious features. It follows that all gastric ulcers should be biopsied with an appropriate number of samples (at least 4 ) reflecting the ulcer size. The number of biopsies taken was not associated with the likelihood of malignant disease in this study, but a number of studies have suggested that 3 to 4 biopsy samples are usually sufficient to diagnose cancer in gastric ulcers with $95 \%$ sensitivity $[12,13]$. The sensitivity reached $100 \%$ when 7 samples were obtained [13]. If biopsies cannot be performed on index endoscopy (because of anticoagulation, gastrointestinal bleeding, etc), a repeat procedure for biopsies should be scheduled at the earliest opportunity to avoid delays in a potential diagnosis of neoplasia.

This study also highlighted that for many patients, FU-OGD was deemed inappropriate due to comorbidities and patient frailty. This is also reflected in the number of patients who died before a follow-up procedure was due. Gastric ulcers are often found in patients who have acute severe illnesses or are elderly with multiple medical issues. In such circumstances the need for FU-OGD needs to be balanced against overall life expectancy and the potential suitability for definitive treatment if a gastric malignancy were found. The high non-attendance rate noted in this study may relate to the uncomfortable and invasive nature of endoscopy. Furthermore patients may have not have been made aware of the reasons for FU-OGD and the implications of non-attendance.

We have demonstrated in this study that early FU-OGD for biopsies in those with no initial biopsies can be achieved in a very busy endoscopy department. Unfortunately many patients waited longer than the recommended 8 weeks after initial benign biopsies for a FU-OGD. However, the cancer yield of FUOGD after benign biopsies remains low. Strategies to reduce the burden of FU-OGD for both patients and the health service would therefore be highly desirable.

We have demonstrated that several simple factors collected during index endoscopy and ulcer biopsy can predict benign disease. Risk stratification using the endoscopist's impression of the gastric ulcer and initial biopsy identified all cases of neoplasia in this study. It therefore may be that the need for FU-OGD in all patients with benign endoscopy and histology should be reconsidered. These data are supported by experience from another center [5] and suggest that the need for FU-OGD may not need to be a blan- 
ket approach for all gastric ulcers but would benefit from evaluation of individual patient risk factors.

There are a number of limitations to our study. While this is a large study, all of the patients came from 1 geographical area in Northern England. Case identification via the endoscopy reporting system allowed capture of all cases of gastric ulcers as these were entered prospectively on the reporting system. Data collection, however, used retrospective methodology by examining endoscopy reports and medical and histology records. Consequently we were unable to examine the influence of other presenting features, particularly weight loss. Furthermore the endoscopist's overall impression of a gastric ulcer was taken from endoscopists' comments in the report's free comment boxes. This method introduces a large degree of subjectivity, but the use of free text is a matter of routine in endoscopy reporting practice. Lesion identification and characterization is a key part of endoscopy training, yet such training often varies in different institutions. It is therefore possible that the practice of endoscopists may vary between different locations. Importantly and in contrast to other studies, this one did not rely on the impression of tertiary referral expert endoscopists. This highlights that endoscopic interpretation by any JAG-certified endoscopist was sufficiently accurate for differentiation between suspicious and non-suspicious appearance.

This study is also limited in that a number of risk factors for gastric cancer could not be addressed. Ethnicity can influence the risk of gastric cancer and Japanese race is associated with an increased risk $[14,15]$. Our study did not record the patient's ethnicity. However, fewer than 37,000 people of Japanese origin reside in the UK ( $0.06 \%$ of the general population) [16] and it is unlikely that Japanese ethnicity would have influenced our results. $H$ pylori is an independent risk factor for gastric malignancy $[17,18]$. Unfortunately, $\mathrm{H}$ pylori status was not documented for some patients and our results should be interpreted with caution with regard to that status. Other risk factors such as atrophic gastritis were not included in our analysis as no biopsies were obtained from non-ulcerated areas of the stomach in accordance with local histopathology guidelines. Furthermore no data on medication usage especially on Aspirin or nonsteroidal anti-inflammatory drugs were available.

For this study the authors chose to report a combined outcome of dysplasia and malignancy. In clinical practice it is of utmost importance for the endoscopist to differentiate benign from potentially malignant (ie dysplastic) and malignant disease. Hence differentiation between clearly benign and the combined outcome of dysplastic/neoplastic disease was considered clinically more relevant. The number of dysplasia cases was too small to allow for separate analysis. The study identified predictors of benign versus malignant disease (rather than malignant versus benign) and therefore promotes the identification of benign disease which may not require close follow-up.Ideally all of our patients should have undergone FU-OGD but it was important to report on the full cohort rather than just selecting those with FU-OGD. The authors included patients without FU-OGD who survived more than 360 days without a cancer diagnosis in the group of patients with benign disease. Given the fast-growing nature of upper gastrointestinal malignancies, the authors opine that this is a clinically justified characterization. Because this group of patients differed from those with FU-OGD only marginally (the numeric differences in number of biopsies obtained are of doubtful clinical importance), that is unlikely to influence the outcome of our analysis. In Japanese cohorts very early-stage cancers and dysplasia may be slowly progressive and it would be possible, but unlikely, to have missed such cases through the 360-day follow-up period [19]. However, all our patients presented with symptoms in the first instance, whereas in Japanese patients, detection was usually through screening and they would have been asymptomatic. Because median survival from gastric cancer is unfortunately only 35 weeks in the United Kingdom [20], the choice of 360-day follow-up is very unlikely to influence the outcomes or our conclusions.

This study has demonstrated a $6 \%$ combined dysplasia and malignancy risk in gastric ulcers. Biopsies of all gastric ulcers should be performed as a matter of routine and, if not possible, at time of first endoscopy, should be scheduled as soon as possible afterwards. However, routine FU-OGD may not be required for all patients with gastric ulcers, as a combination of benign endoscopy appearance and benign histology accurately predicted benign disease in this study.

\section{Competing interests: None}

\section{References}

1 Kuipers EJ, Thijs JC, Festen HP. The prevalence of Helicobacter pylori in peptic ulcer disease. Aliment Pharmacol Ther 1995; 9: (Suppl 02) 5969

2 Bustamante M, Devesa F, Borghol A et al. Accuracy of the initial endoscopic diagnosis in the discrimination of gastric ulcers: is endoscopic follow-up study always needed? J Clin Gastroenterol 2002; 35: 25 - 28

$3 \mathrm{Lv} \mathrm{SX}$, Gan JH, Ma XG et al. Biopsy from the base and edge of gastric ulcer healing or complete healing may lead to detection of gastric cancer earlier: an 8 years endoscopic follow-up study. Hepatogastroenterol 2012; 59: $947-950$

4 Amorena Muro E, Borda Celaya F, Martínez-Peñuela Virseda JM et al. [Analysis of the clinical benefits and cost-effectiveness of performing a systematic second-look gastroscopy in benign gastric ulcer]. Gastroenterol Hepatol 2009; 32: 2-8

5 Mañas MD, Domper A, Albillos A et al. Endoscopic follow-up of gastric ulcer in a population at intermediate risk for gastric cancer. Rev Esp Enferm Dig 2009; 101: 317 - 324

6 National Institue for Health and Care Excellence. Dyspepsia: Management of dyspepsia in adults in primary care. In: excellence NIfHaC (ed) NICE clinical guideline 17; 2004

7 National Institue for Health and Care Excellence. Dyspepsia and gastrooesophageal reflux disease. NICE clinical guideline 184; 2014

8 Banerjee S, Cash BD, Dominitz JA et al. The role of endoscopy in the management of patients with peptic ulcer disease. Gastrointest Endosc 2010; 71: $663-668$

9 Uemura N, Sugano K, Hiraishi $H$ et al. Risk factor profiles, drug usage, and prevalence of aspirin-associated gastroduodenal injuries among high-risk cardiovascular Japanese patients: the results from the MAGIC study. J Gastroenterol 2014; 49: 814-824

10 [Anonymous]. Defining Research. In: Health Research Authority; London: 2009

11 Schlemper RJ, Riddell RH, Kato $Y$ et al. The Vienna classification of gastrointestinal epithelial neoplasia. Gut 2000; 47: 251 - 255

12 Choi Y, Choi HS, Jeon WK et al. Optimal number of endoscopic biopsies in diagnosis of advanced gastric and colorectal cancer. J Korean Med Sci 2012; 27: 36-39

13 Graham DY, Schwartz JT, Cain GD et al. Prospective evaluation of biopsy number in the diagnosis of esophageal and gastric carcinoma. Gastroenterology 1982; 82: 228-231

14 Naylor GM, Gotoda T, Dixon $M$ et al. Why does Japan have a high incidence of gastric cancer? Comparison of gastritis between UK and Japanese patients Gut 2006; 55: $1545-1552$

15 Namekata T, Miki K, Kimmey M et al. Chronic atrophic gastritis and Helicobacter pylori infection among Japanese Americans in Seattle. American journal of epidemiology 2000; 151: 820-830

16 [Anonymous]. 2011 Census: Population Estimates for the United Kingdom. In: Statistics OfN (ed). London: 2011 
17 Yeh JM, Ho W, Hur C. Cost-effectiveness of endoscopic surveillance of gastric ulcers to improve survival. Gastrointest Endosc 2010; 72: 33 43

$18 W u$ CY, Kuo KN, Wu MS et al. Early Helicobacter pylori eradication decreases risk of gastric cancer in patients with peptic ulcer disease. Gastroenterology 2009; 137: 1641 - 1648
19 Tsukuma H, Oshima A, Narahara $H$ et al. Natural history of early gastric cancer: a non-concurrent, long term, follow up study. Gut 2000; 47: $618-621$

20 Devane C. Living after diagnosis: Median cancer survival times. In: Macmillan Cancer Support as accessed on 13/01/2016. http://www. macmillan.org.uk/Documents/AboutUs/Newsroom/LivingAfterCancerMedianCancerSurvivalTimes.pdf 